\title{
The Correlation of the Urban Images
}

\author{
Hayat Bendahri \\ University of Abdelmalek \\ Essaadi Morocco,National \\ school of the Sciences Applied \\ of Tangier (ENSAT) Laboratory \\ of Information Technologies \\ and the Communication \\ (LabTIC)
}

\author{
Abdelhak Ezzine \\ University of Abdelmalek \\ Essaadi Morocco,National \\ school of the Sciences Applied \\ of Tangier (ENSAT) Laboratory \\ of Information Technologies \\ and the Communication \\ (LabTIC)
}

\author{
Otman Filali Meknassi \\ University of Abdelmalek \\ Essaadi Morocco,National \\ school of the Sciences Applied \\ of Tangier (ENSAT) Laboratory \\ of Information Technologies \\ and the Communication \\ (LabTIC)
}

\begin{abstract}
In this paper, we present a demonstration for the urban images to prove the existence of the redundancy of information on three spectral bands. The thing that we can notice it visually and proven thanks to a statistical study carried out on the urban image. The two treatments (visual, statistical) assure us that the images of the road have a strong correlation
\end{abstract}

\section{Keywords}

Correlation, RGB space color, Lab space color, correlation coefficient.

\section{INTRODUCTION}

The urban image is considered in each system as a threedimensional statistical distribution (1). Generally, the three data components natural images are always more or less correlated whaterver the space of representation. A space of the color is a manner of representing the colors and their relations between them (2). Among these spaces of the color (3), we find RGB and Lab that well be used in the study. Initially, we will try to show the relation between the various chromatic channels exposing urban images.

With the intension, we presented a visual and statistical approach. The visual part rests on the observation of an image projected on the three chromatic channels. Whereas, the statistical part consists in calculating the centered normalized correlation coefficient between the chromatic bands. If two variables are related in the sense that change in any one variable is accompanied by change in the other [4]. This study is justified by the analysis of various experimental results which show the strong relation between the various axes in each space of color (RGB and Lab).

The remaining of the paper is organized as follows. Section 2 describes the notion of the correlation. Section 3 shows the correlation between the bands of RGB space. Section 4 shows the correlation between the channels of Lab space. Section 5 describes our contribution and the steps affecting this and shows the result, and in section 6 , there is a conclusion and future research.

\section{CORRELATION FUNCTION}

\subsection{Definition}

In statistical theory [5], correlation is a statistical measure that indicates the extent to which two or more variables fluctuate together. A measurement of this correlation is obtained by the calculation of the linear correlation coefficient "Bravais Pearson".

The correlation coefficient of two variables in a data sample is their covariance divided by the product of their individual standard deviations. It is a normalized measurement of how the two are linearly related.
Formally, the sample correlation coefficient is defined by the following formula, where $\sigma_{x}$ and $\sigma_{y}$ are the population standard deviations, and $\operatorname{cov}(\mathrm{X}, \mathrm{Y})$ is the sample covariance.

$$
\operatorname{cor}(X, Y)=\frac{\operatorname{cov}(X, Y)}{\sigma_{X} \sigma_{Y}}
$$

A positive correlation indicates the extent to which those variables increase or decrease in parallel; a negative correlation indicates the extent to which one variable increases as the other decreases. We proceed by two treatments, the first rests on the treatments by line, and the second for treatment by column.

A perfect correlation of (-/+) 1 occurs only when the data points all lie exactly on a straight line. If $r=+1$, the slope of this line is positive. If $r=-1$, the slpoe of this line is negative.

A correlation [6] greater than 0.8 is generally described as strong, whereas a correlation less than 0.5 is generally described weak.

\subsection{Matrix of Correlation}

A matrix $\mathrm{R}$ of correlation coefficients calculated from an input matrix $\mathrm{X}$ whose rows are observations and whose columns are variables. The matrix $\mathrm{R}$ is related to the covariance matrix $\operatorname{cov}(X)$ by:

$$
R\left(X_{i}, X_{j}\right)=\frac{\operatorname{cov}\left(X_{i}, X_{j}\right)}{\sqrt{\operatorname{cov}\left(X_{i}, X_{i}\right) \operatorname{cov}\left(X_{j}, X_{j}\right)}}
$$

\section{CORRELATION BETWEEN THE CHANNELS OF RGB SPACE}

To show this correlation binding the three color axis, we propose an algorithm which functions as following:

- $\quad$ To take an RGB image;

- To extract its three chromatic matrices;

- To visualize the image on the three chromatic axis

- To calculate the correlation coefficient between the channels chromatic R,G,B.

The following figures expose the strong correlation between the three chromatic axes representing our image

The figure 1 for the visual part, whereas figures 2 and 3 for the statistical part:

\subsection{Visual Part}

Though the figure 1 , we present the projection of the urban image on the three plans, we notice the redundancy of the information on three plans, to confirm this result; we proceed by a statistical study. 


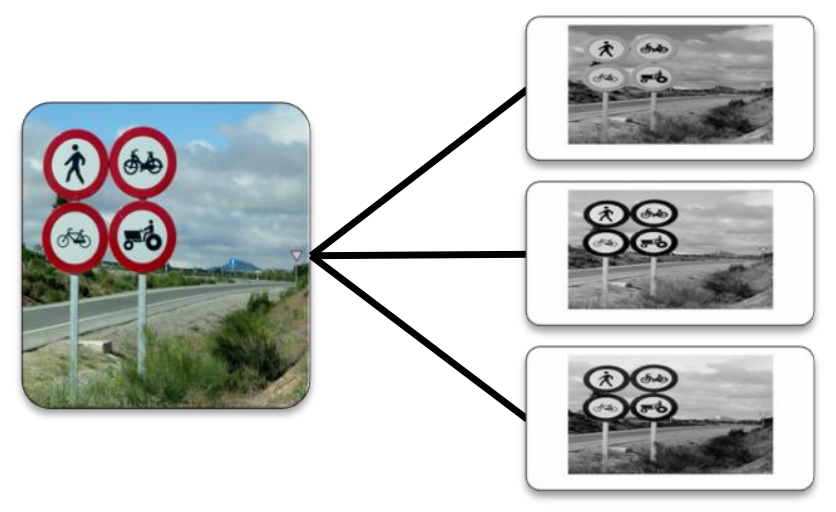

$\mathrm{R}$

G

B

\subsection{Statistical Part}

In this part, we present another treatment consists in calculating the centered normalized correlation coefficient between the chromatic bands, and confirm the results obtained in the visual study.

\begin{tabular}{|l|l|l|l|}
\hline & Coefficient RG & Coefficient RB & Coefficient GB \\
\hline Image 1 & 0,999018534588985 & 0,991591347099429 & 0,996318886493589 \\
\hline Image 2 & 0,998576545669545 & 0,992383337569015 & 0,997331442574078 \\
\hline Image 3 & 0,977378312874473 & 0,977378312874473 & 0,974943182338106 \\
\hline
\end{tabular}

Figure 2: Table of coefficient of correlation for the lines

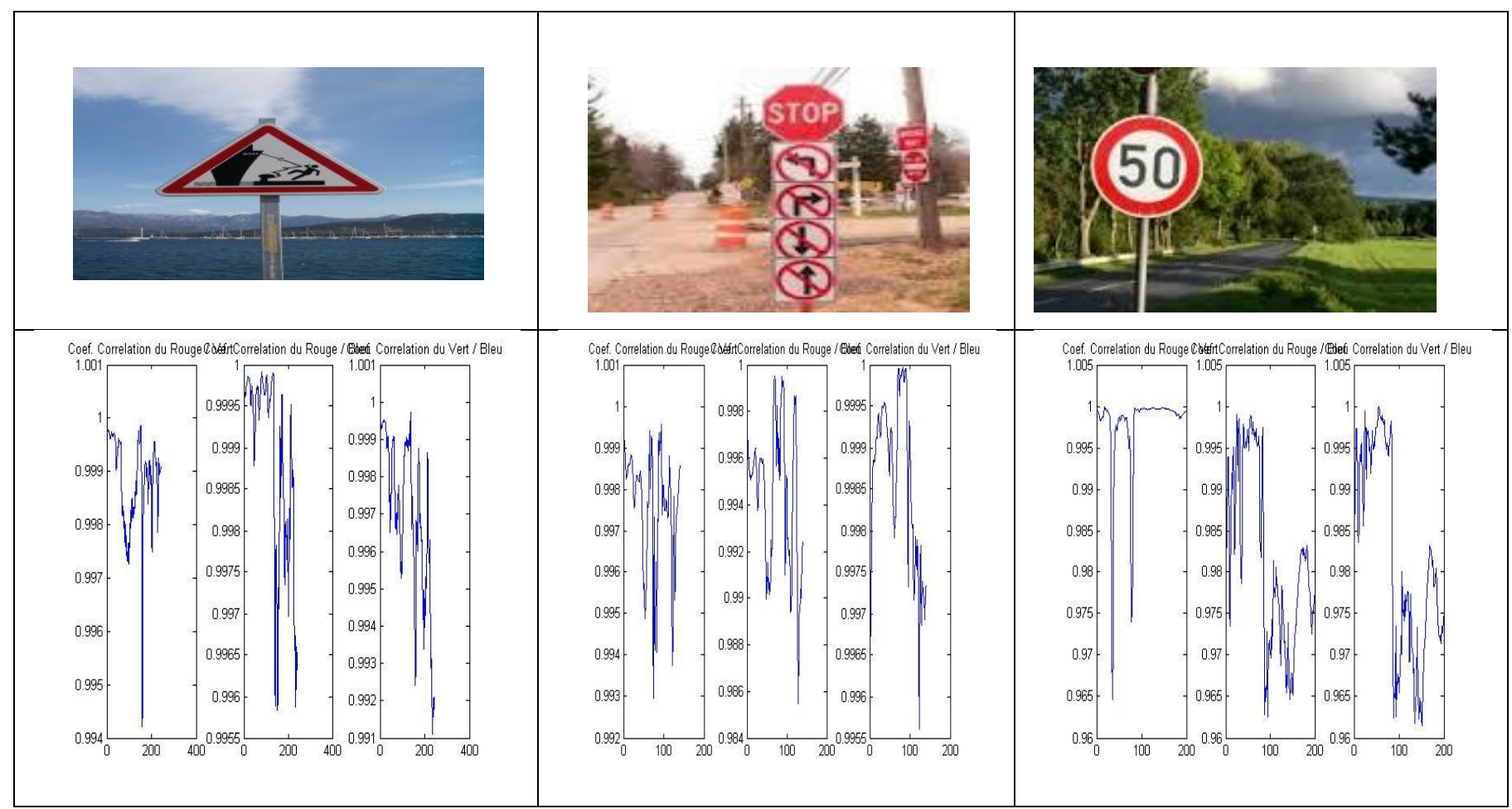




\subsubsection{Treatment by column}

We reaffirm the results already seen with same manner. In the same way, the results mentioned through the table of coefficient of correlation for the columns (see figure 3),

\begin{tabular}{|l|l|l|l|l|}
\hline & Coefficient RG & Coefficient RB & Coefficient GB \\
\hline $\begin{array}{l}\text { Image } \\
1\end{array}$ & 0,998644391393148 & 0,996821566363540 & 0,991355896181445 \\
\hline $\begin{array}{l}\text { Image } \\
2\end{array}$ & 0,999855895941741 & 0,998881595597865 & 0,999136236719117 \\
\hline $\begin{array}{l}\text { Image } \\
3\end{array}$ & 0,993572849668524 & 0,960975850938847 & 0,938173133197139 \\
\hline
\end{tabular}

Figure 3: Table of coefficient of correlation for the columns

According the experiments carried out; visual (projection the image on the three chromatic channels), statistical (calculate the correlation coefficient between the spectral bands), on a database of urban images test. It is concluded that the channels R,G,B of RGB space are strongly correlated, whether it is on the lines or columns. We enclose our treatment by the confirmation of the existence of a heavy correlation for the natural images presented on the RGB space.

\section{CORRELATION BETWEEN THE CHANNELS OF Lab SPACE}

To show this correlation binding the three color axis, we propose an algorithm which functions as following:

- $\quad$ To take an RGB image; validate the existence of a strong correlation between the spectral bands in the RGB color space.
- To convert this image in Lab space;

- To extract its three chromatic matrices;

- To visualize the image on the three chromatic axis

- To calculate the correlation coefficient between the channels chromatic L,a,b.

The results are presented in the following figures, see figure 4 for the visual part and figure 5 for the statistical part.

\subsection{Visual part}

The figure shows the redundancy of information on the three plans, because we find in the three plans the same details. So, repetition of information, that means by correlation between these bands L,a,b.

L

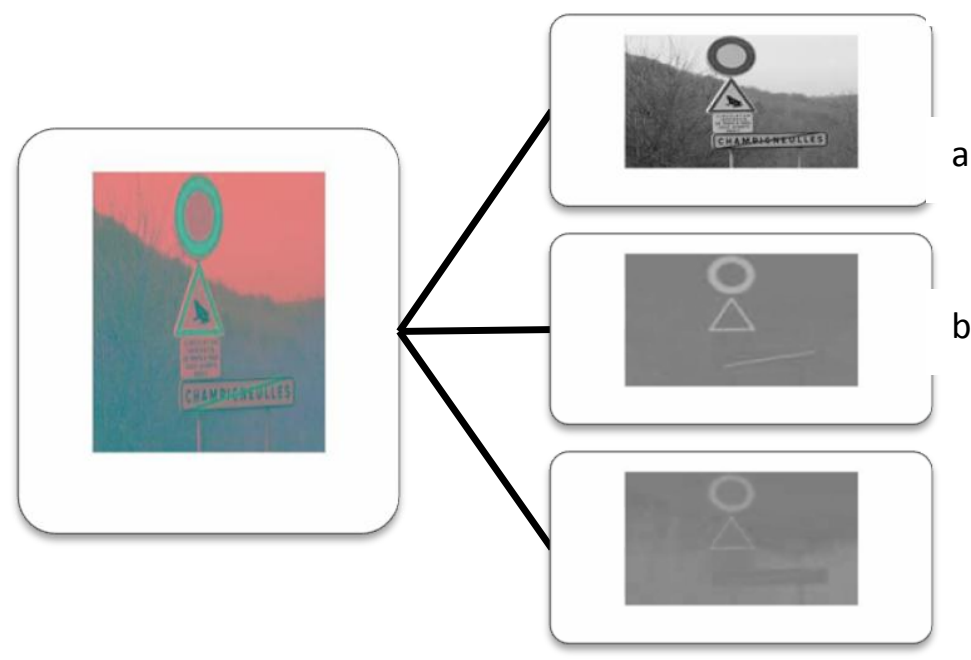

Figure 4 : visualization of the imageon the three plans L,a,b 


\subsection{Statistical part}

\subsubsection{Treatment by line}

\begin{tabular}{|l|l|l|l|l|}
\hline & Coefficient La & Coefficient Lb & Coefficient ab \\
\hline $\begin{array}{l}\text { Image } \\
1\end{array}$ & 0,859396573590786 & 0,843667395244030 & 0,999519436810661 \\
\hline $\begin{array}{l}\text { Image } \\
2\end{array}$ & 0,882919991326285 & 0,864742756406686 & 0,999260900220385 \\
\hline $\begin{array}{l}\text { Image } \\
3\end{array}$ & 0,992276290263916 & 0,993882615528022 & 0,999906150587120 \\
\hline
\end{tabular}

Figure 5: Table of coefficient of correlation for the lines

\subsubsection{Treatment by column}

\begin{tabular}{|l|l|l|l|}
\hline & Coefficient La & Coefficient Lb & Coefficient ab \\
\hline \begin{tabular}{l|l|l|l|}
\hline Image \\
1
\end{tabular} & 0,722271351942677 & 0,768338255656688 & 0,994306032481846 \\
\hline $\begin{array}{l}\text { Image } \\
2\end{array}$ & 0,888179063551371 & 0,888179063551353 & 0,999999999999997 \\
\hline $\begin{array}{l}\text { Image } \\
3\end{array}$ & 0,603752334900524 & 0,625330424941544 & 0,998994639759409 \\
\hline
\end{tabular}

Figure 6: Table of coefficient of correlation for the columns

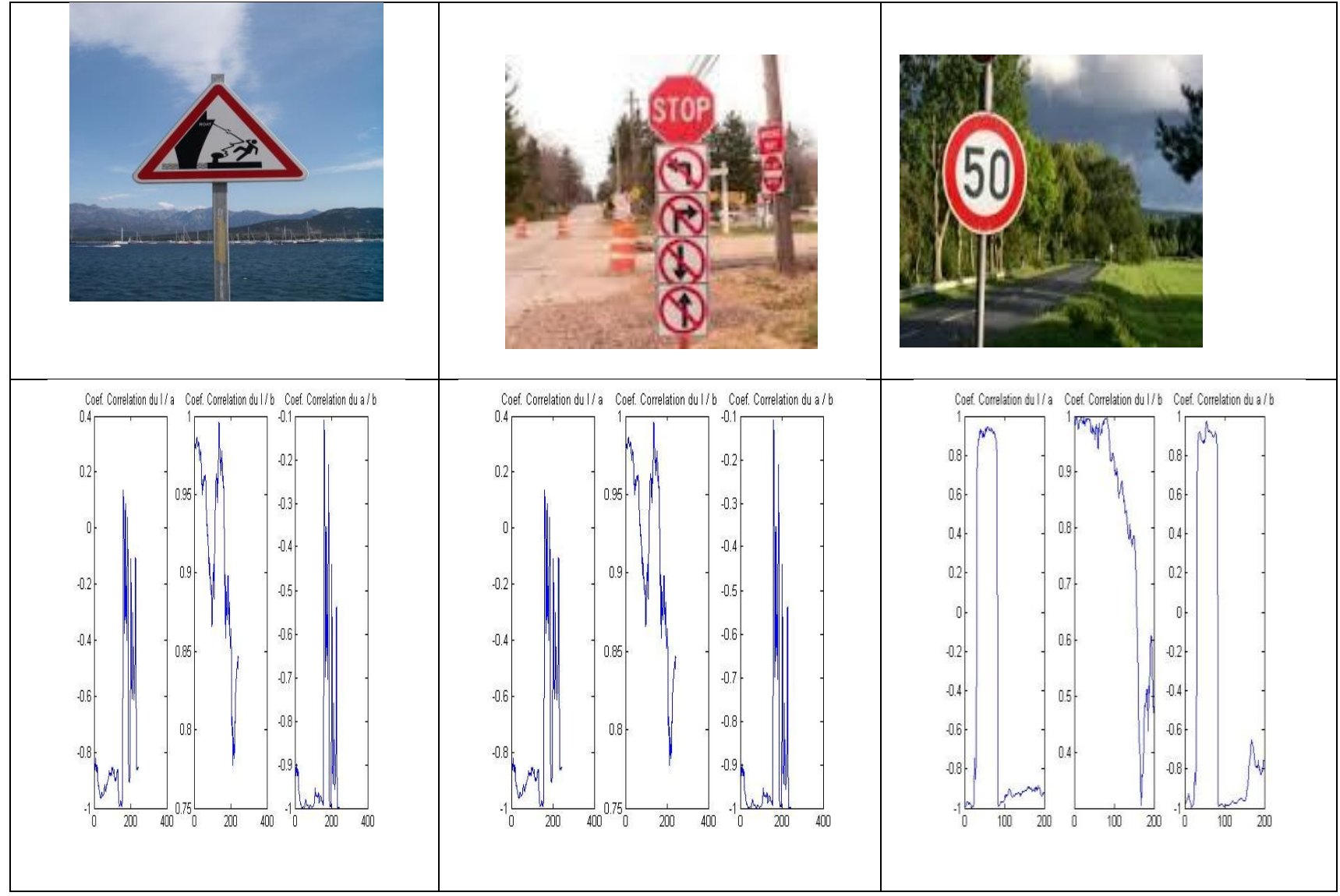

Figure 7: Analysis of the correlation between the various lines of the chromatic channels of the color space Lab 
However it is noted that the variations observed are of less degree compared to the space of RGB space what confirms that Lab space is correlated less.

\section{CONCLUSION}

We deduce from these sections that the various channels of space RGB are strongly correlated. While in the space Lab which is space with constant chromaticity only channels a and $\mathrm{b}$ for Lab space. We note that space Lab is less correlated than RGB space. Seven images were used for these statistical tests. The found results are summarized in the following table:

\begin{tabular}{|c|c|c|c|c|c|c|c|c|}
\hline & $\begin{array}{c}\text { Coe } \\
\mathrm{f}\end{array}$ & $\begin{array}{c}\text { Image } \\
1\end{array}$ & $\begin{array}{c}\text { Image } \\
2\end{array}$ & $\begin{array}{c}\text { Image } \\
3\end{array}$ & $\begin{array}{c}\text { Image } \\
4\end{array}$ & $\underset{5}{\text { Image }}$ & $\underset{6}{\text { Image }}$ & $\begin{array}{c}\text { Image } \\
7\end{array}$ \\
\hline \multirow{3}{*}{$\begin{array}{c}\mathrm{RG} \\
\mathrm{B}\end{array}$} & $\mathrm{RG}$ & 0.999 & 0.999 & 0.999 & 0.999 & 0.999 & 0.999 & 0.999 \\
\hline & $\mathrm{RB}$ & 0.999 & 0.999 & 0.999 & 0.999 & 0.997 & 0.998 & 0.997 \\
\hline & $\mathrm{BG}$ & 0.999 & 0.999 & 0.998 & 0.999 & 0.998 & 0.998 & 0.999 \\
\hline \multirow{3}{*}{ Lab } & $\mathrm{La}$ & 0.72 & 0.80 & 0.6 & 0.88 & 0.80 & 0.86 & 0.8 \\
\hline & $\mathrm{Lb}$ & 0.76 & 0.806 & 0.62 & 0.88 & 0.80 & 0.86 & 0.95 \\
\hline & $a b$ & 0.9 & 0.96 & 0.97 & 0.9 & 0.96 & 0.8 & 0.8 \\
\hline
\end{tabular}

Figure 8: correlation coefficient for two sapce LAb and RGB

In the natural conditions, the problem of road signs detection has an obstacle of the correlation between the various colors of the image. Generally, the images of the same natural scene recorded according to the various spectral bands of the sensor are highly correlated. In order to stage this problem, we proceed by a statistical study, the reason which pushes us, in what follows, to regard the image as a three dimensional statistical distribution. Within this framework we try to think of a technique which will help us to answer the key question "how can remove this correlation?", or how let us can associate with our image such an adequate spectral representation which enables us to work under the suitable conditions to extract in a reliable way and specifies the control signs road having in the image? This is why, we resort to techniques in general the data analysis. Those can be used for to cure this problem of correlation between the components, as Principal Component Analysis or Independent component analysis [8]. We proceed by PCA, Principal Component Analysis is a dimension-reduction tool that can be used advantageously in such situations. Principal component analysis aims at reducing a large set of variables to a small set that still contains most of the information in the large set.

\section{REFERENCES}

[1] Color image processing and applications. Plataniotis, K. N., \& Venetsanopoulos, A. N. s.l. : springer, 2000.

[2] Vandenbroucke, N., \& Macaire, L. les espaces de la couleur.

[3] Vandenbrouke, Nicola. Segmentation d'images couleur par classification de pixels dans des espaces d'attributs colorimétriques adaptés. Application à l'analyse d'images de football. Lille: Université des Sciences et Technologies de Lille, (thèse de doctorat) : s.n., 200.

[4] WERT, James E., NEIDT, Charles O., et AHMANN, J. Stanley. . Coefficient of correlation. 1954.

[5] Miller, I., Freund, J. E., \& Johnson, R. A. (1965). Probability and statistics for engineers (Vol. 1110). Englewood Cliffs, NJ: Prentice-Hall.

[6] Al., Benzécri J. -P. et. l'analyse des données. Paris : Dunod, 1976.

[7] Miller, I., Freund, J. E., \& Johnson, R. A. Probability and statistics for engineers . s.l.: Englewood Cliffs, NJ: Prentice-Hall., (1965). (Vol. 1110).

[8] ACP et ACI pour la reduction de données en imagerie astronomique multispectrale. Farid FLITTI1, al. 2003. GRETSI.

[9] Tenenhaus, M. La régression PLS: théorie et pratique. La régression PLS: théorie et pratique. s.l.: Editions Technip, 1998. 